University of Bristol, UK

angela.raffle@bristol.ac.uk Cite this as: BMJ 2020;371:m4438 http://dx.doi.org/10.1136/bmj.m4438 Published: 19 November 2020

\title{
Screening the healthy population for covid-19 is of unknown value, but is being introduced nationwide
}

\author{
Concerns this will see demolition of hard won frameworks for protecting the public from ineffective, \\ poorly delivered, and unethically practised screening
}

\section{Angela E Raffle consultant in public healthhonorary senior lecturer}

On 9September The BMJ reported leaked plans, called "Operation Moonshot," to regularly screen households, cities, and then the entire UK population for SARS-CoV-2, at a reported cost of over €1oobn (€112bn; \$132bn). ${ }^{1}$ This appeared to be one of the most unethical proposals for use of public funds, or for screening, that I'd ever seen. Condemnation followed, together with a legal challenge by the Good Law Project. ${ }^{2}$ The UK National Screening Committee (NSC)-the recognised source of expert, independent, and conflict free advice to ministers on screening-had played no part in the decision making process, and the legality of the procurement processes have been questioned.

Subsequently it was reported that the UK government had abandoned plans for Operation Moonshot. Yet, on Tuesday 3 November Moonshot phase I began in Liverpool. According to the city council's website "everyone who lives or works in the city" is eligible for regular and repeat testing and can attend one of 14 army run test centres over the course of two weeks. ${ }^{3}$ The website says this is the beginning of a national roll out and "will help to demonstrate that massive asymptomatic testing can identify far more cases and break the transmission of coronavirus." The army is conducting testing for 11 to 18 year olds in schools, and a letter to parents from one school head advised "if you wish to exclude your child from this test please do so in writing by first thing on Monday morning." Screening for under $11 \mathrm{~s}$ is not mentioned. Official communication to residents has been through news announcements, appealing to public duty and claiming that the test detects "infectiousness." At the time of writing (8 November) Public Health England were keeping the results of its studies on the accuracy of the test confidential. These were subsequently released on 12 November. No information has been given to participants to explain whether the project is actually research, or how personal data will be held and used. An assumption is made that everyone has a smart phone.

A letter to Liverpool MPs on 5 November asking for information about the decision making processes, the nature of the project, informed consent, and data protection safeguards yielded assurance from university staff that "analysis of the pilot is being conducted by the University of Liverpool and a national team of academic collaborators," but specific answers to the questions were not given. ${ }^{4}$

These events worry me. I have been involved in screening since 1984; running major screening programmes, helping set up the UK National
Screening Committee in 1996, and publishing on screening outcomes, research methodology, policy making, delivery, ethics, quality assurance, training, and economics. ${ }^{5}$ Many people think that screening is a simple, straightforward approach to prevention, yet experience has taught us that it is counterintuitive, complex, and paradoxical. Since the 1980 s I have seen, and helped make happen, a transformation of UK screening from a haphazard mess of some 300 unevaluated activities causing untold harm, into the UK National Screening Programmes comprising 11 systematic and evidence based national programmes which screen for 36 different conditions, all regularly publishing data on good performance.

A key lesson is that you must not assume that screening will achieve the outcome you think it will. Observational studies claim to "show" a benefit because people turn up and you find positive cases, but once a well designed control trial takes place the real balance of benefit and harm often proves different. What matters is not just test performance, but the full consequences, desired, unintended, and collateral, of the entire system. At best the Liverpool project can only tell us who comes forward; how many test positive, negative, or unsatisfactory; how rapid tests compare with polymerase chain reaction tests (if both are done); and some measure of compliance with advice to self-isolate. It cannot tell us how many cases will be found that are truly infectious and that would not otherwise have come to light through well performed contact tracing. Nor can it tell us whether identification through screening caused a significant behaviour change, either ahead of or in the absence of symptoms, resulting in a worthwhile change to transmission. Without seeing the research protocol we don't know whether the pilot will measure undesired outcomes. These will include false reassurance for infectious or about-to-be-infectious people who test negative, unnecessary isolation in non-infectious people who test positive, trauma or distress from the testing process especially in children, and massive opportunity cost from diversion of material and intellectual resources away from other more beneficial uses.

Ethical matters in screening are complex, and it took fierce campaigning from the 1960 s onwards for these to be recognised in GMC ethics advice and through the NSC's own Ethics Task Group. Moonshot seems to be taking us back to the paternalistic 1960s, potentially breaching the Helsinki Declaration and ignoring data protection and privacy laws. ${ }^{6-9}$ 
We may be in the middle of a pandemic, but this is no reason to lose our heads. All the documentation about decision making for the Liverpool project needs to be brought into the public domain for independent scrutiny, and we need the NSC to prepare evidence based advice to ministers on the Moonshot proposals. The history of UK screening has seen phases of blind optimism (1960s), deep disillusionment (1970s), transformational organisation (1980s), increased professional understanding (1990s), and relentless commercially driven pressure for unevaluated screening and direct-to-consumer promotion (200os onwards). My fear is that the 2020s will see demolition-with Moonshot as the wrecking ball-of hard won frameworks for protecting the public from ineffective, poorly delivered, and unethically practised screening, and from the consequences of non-evidence based use of public funds. ${ }^{10}$ We must be awake to the dangers.

Angela Raffle has 35 years of experience of improving health screening, working for the UK National Screening Programmes since their inception in 1996, and is lead author of the leading international postgraduate textbook Screening: Evidence and Practice (2019).

Competing interests: AR delivers training and education for the UK National Screening Programmes.

Commissioned, not peer reviewed

1 lacobucci G, Coombes R. Covid-19: Government plans to spend $f 100 \mathrm{bn}$ on expanding testing to 10 million a day. BMJ2020;370:m3520. doi: 10.1136/bmj.m3520 pmid: 32907851

2 Deeks JJ, Brookes AJ, Pollock AM. Operation Moonshot proposals are scientifically unsound. BMJ 2020;370:m3699. doi: 10.1136/bmj.m3699 pmid: 32963111

3 Liverpool City Council. Everyone can get a free covid-19 test now. 2020. https://iverpool.gov.uk/covidtesting.

4 Pollock A. Letter to Liverpool MPs. 5 November 2020. https://allysonpollock.com/?page_id=3345.

5 Raffle AE, Mackie A, Gray JAM. Screening: Evidence and Practice. 2nd ed. Oxford University Press, 2019doi: 10.1093/med/9780198805984.001.0001.

6 Raffle AE, Gray JAM. The 1960s cervical screening incident at National Women's Hospital, Auckland, New Zealand: insights for screening research, policy making, and practice. J Clin Epidemiol 2020;122:A8-13. doi: 10.1016/j.jclinepi.2020.04.008 pmid: 32448444

7 Raffle AE. Information about screening - is it to achieve high uptake or to ensure informed choice? Health Expect 2001;4:92-8. doi: 10.1046/j.1369-6513.2001.00138.x pmid: 11359539

8 General Medical Council. Consent: patients and doctors making decisions together. 2008. www.gmc-uk.org/static/documents/content/Consent__English_0617.pdf.

9 World Medical Association. Declaration of Helsinki: ethical principles for medical research involving human subjects. July 2018. www.wma.net/policies-post/wma-declaration-of-helsinki-ethicalprinciples-for-medical-research-involving-human-subjects.

10 Sturdy S, Miller F, Hogarth S, et al. Half a century of Wilson and Jungner: reflections on the governance of population screening. Wellcome Open Res 2020;5:158.

doi: 10.12688/wellcomeopenres.16057.2 pmid: 32923689 